\title{
A UTILIZAÇÃO DO MÉTODO NODAL NA SIMULAÇÃ̃O DE PROCESSOS TÉRMICOS
}

\author{
C. R. RODRIGUES VELOSO ${ }^{1}$, R. GEDRAITE ${ }^{2}$ \\ ${ }^{1}$ Bolsista PIBIC FAPEMIG/UFU, discente do curso de Engenharia Química \\ 2 Professor da Faculdade de Engenharia Química da UFU/MG \\ E-mail para contato: rgedraite@feq.ufu.br
}

\begin{abstract}
RESUMO - No processamento térmico de produto alimentício industrializado é muito importante que a temperatura do mesmo alcance valores adequados e suficientes para assegurar um índice de letalidade pré-estabelecido a ser imposto ao microrganismo de referencia. Tipicamente, a determinação desta temperatura é feita de maneira experimental por meio de testes de penetração de calor no produto considerado. Neste trabalho se estudou o desenvolvimento de modelo matemático discretizado, desenvolvido com base na nodalização do volume de controle adotado, obtido a partir das equações de balanço global de energia e de transporte de calor que permita representar de forma adequada o comportamento temporal da temperatura do sistema estudado. O modelo matemático obtido foi validado por comparação com dados disponíveis na literatura. Com base no modelo obtido, foi estudada influência da propriedade difusividade térmica sobre o comportamento da temperatura simulada. Os resultados obtidos mostraram que o desvio entre os valores de temperatura medidos experimentalmente e preditos pelo modelo é afetado pelo valor da propriedade de transporte de calor considerada neste trabalho e, também, pela posição do nó considerado na análise.
\end{abstract}

\section{INTRODUÇÃO}

O processamento térmico de alimentos industrializados consiste no aquecimento de embalagens em autoclaves pressurizadas, que podem ser estacionárias ou rotativas, verticais ou horizontais, e/ou contínuas ou descontínuas. O processamento térmico deve ser especificado por especialistas que, para cada sistema equipamento/embalagem/alimento definem as condições de processo: tempo de processo, temperatura de processo, temperatura mínima inicial do alimento, perfil de pressão e programa de desaeração da autoclave.

Em face destes requisitos de qualidade e de segurança, grande cuidado é tomado no cálculo destes tempos de processamento, assim como no controle do tempo e da temperatura vigentes durante os mesmos, para evitar que o alimento seja submetido a um subprocessamento ou a um superprocessamento (Germer, 1997).

O objetivo deste trabalho é apresentar um modelo matemático, desenvolvido para ser executado em tempo real, e que leve em consideração as condições de operação de uma autoclave estacionária. Este modelo poderá ser empregado em conjunto com o sistema de controle da autoclave, com a finalidade de garantir o nível de letalidade previamente estabelecido para micro-organismos. 


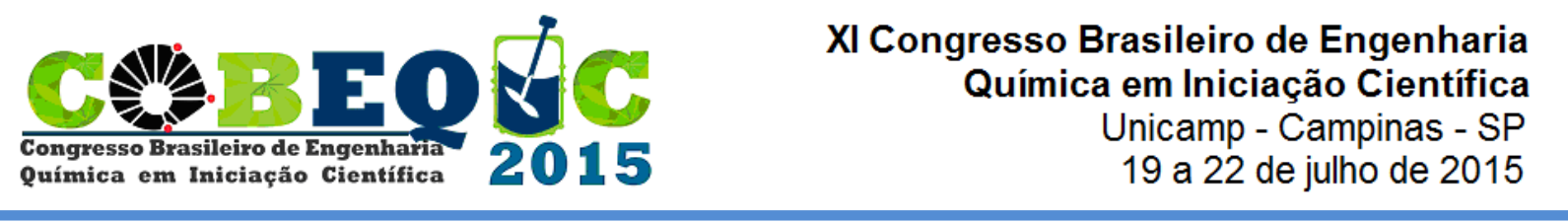

\section{DESCRIÇÃO DO PROBLEMA}

A esterilização de um produto alimentício depende, em parte, da taxa de penetração de calor no mesmo.

Quando produtos alimentícios são colocados numa embalagem, que por sua vez é colocada numa autoclave e processada termicamente através da condensação do vapor de água saturado, a taxa de transferência de calor para os mesmos depende dos seguintes fatores (Teixeira; Balaban, 1996):

a) Coeficientes de transferência de calor;

b) Propriedades físicas do produto alimentício e da embalagem;

c) Diferença de temperatura entre o produto alimentício e autoclave; e

d) Tamanho da embalagem.

O foco neste trabalho foi a predição da evolução do perfil de temperaturas no interior de uma embalagem metálica de geometria cilíndrica contendo proteína texturizada de soja (PTS).

O produto usado no trabalho experimental desenvolvido por Gedraite et al, (1998) foi obtido pelo processamento da PTS em instalação industrial localizada em São Paulo/SP. Nos ensaios experimentais realizados empregou-se embalagem metálica cilíndrica do tipo $300 \mathrm{X}$ 406 (73,3 mm X $111 \mathrm{~mm})$.

\section{CONTROLE DO PROCESSO DE ESTERILIZAÇÃO EM TEMPO REAL}

O emprego de modelos matemáticos capazes de predizer as características do produto alimentício em função de alterações nas condições de processo é fundamental para um bom desempenho dos sistemas de controle baseados em computador que sejam executados em tempo real.

A principal vantagem advinda do uso de modelos matemáticos capazes de estimar o histórico da temperatura do produto é o fato de que se podem aceitar variações na temperatura da autoclave. Assim sendo, na ocorrência de uma diminuição inesperada da temperatura da autoclave, o modelo fornecerá rapidamente o histórico das temperaturas e calculará o valor do nível de letalidade atingido.

Pretende-se neste trabalho que o modelo matemático desenvolvido venha a ser parte integrante de um aplicativo usado para auxiliar a tomada de decisões por um sistema de controle baseado em computador. Assim, na ocorrência de uma perturbação na temperatura da autoclave, o modelo deverá prever a resposta correta da temperatura no interior do produto alimentício para este desvio, bem como o efeito correspondente sobre o fator de letalidade acumulado $\left(\mathrm{F}_{0}\right)$. O modelo matemático deverá, também, gerar informação que permita reprogramar o tempo de processamento, continuando o aquecimento até que a letalidade acumulada alcance o valor pré-estabelecido. 


\section{DESENVOLVIMENTO DO MODELO MATEMÁTICO}

A equação que governa a transferência de calor para a PTS em uma embalagem de altura $\mathrm{H}$ e raio $\mathrm{R}$, com difusividade térmica constante $\alpha$ é dada pela Equação (1).

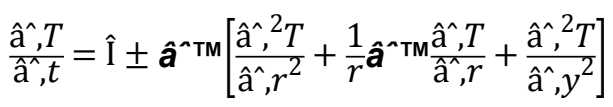

A Figura 1 apresenta uma representação esquemática de uma embalagem cilíndrica típica, que usa as coordenadas anteriormente citadas.

No desenvolvimento deste trabalho, foram consideradas as seguintes hipóteses simplificadoras (Teixeira; Balaban, 1996):

a) A temperatura do produto alimentício é uniforme no instante $t=0$;

b) A temperatura da embalagem cilíndrica é constante depois de $\mathrm{t}=0$ (não há variação entre dois pontos distintos da embalagem);

c) $\mathrm{O}$ coeficiente de transferência de calor, externo à embalagem, é muito elevado (isto é, a resistência térmica da superfície é desprezível);

d) A difusividade térmica não varia com o tempo, com a temperatura, ou espacialmente no interior do produto alimentício; e

e) Não existe headspace no interior da embalagem.

f) O modelo matemático foi desenvolvido tomando-se por base a discretização do conjunto embalagem / produto alimentício em um número finito de elementos de volume, supostos isotérmicos (Gedraite, 1999). Na Figura 2 é apresentada a discretização típica adotada neste trabalho, na qual são mostradas as conexões de um determinado nó com os seus vizinhos.

g) A equação geral de balanço de energia para um determinado nó no qual ocorre a difusão do calor é composta pelo termo de variação temporal da temperatura (lado esquerdo da equação) e pelos termos de troca térmica entre o nó considerado e seus vizinhos, assumindo a hipótese de que não há fonte de calor no interior do produto alimentício (Bastos, 1994). Na Equação (2) é apresentado o balanço global de energia considerado.

$$
C_{I} \hat{\mathrm{a}}^{\wedge \mathrm{rm}} \frac{d T_{I}}{d t}=G_{I N} \hat{\mathrm{a}}^{\wedge \mathrm{TM}}\left(T_{N}-T_{I}\right)+G_{I S} \hat{\mathrm{a}}^{\hat{\mathrm{A}}^{\mathrm{Tm}}}\left(T_{S}-T_{I}\right)+G_{I L} \hat{\mathrm{a}}^{\wedge \mathrm{TM}}\left(T_{L}-T_{I}\right)+G_{I N} \hat{\mathrm{a}}^{\wedge \mathrm{TM}}\left(T_{N}-T_{I}\right)+G_{I O} \hat{\mathrm{a}}^{\wedge \mathrm{TM}}\left(T_{O}-T_{I}\right)
$$

Figura 1 - Representação esquemática de
Figura 2 - Discretização típica do conjunto 
uma embalagem cilíndrica

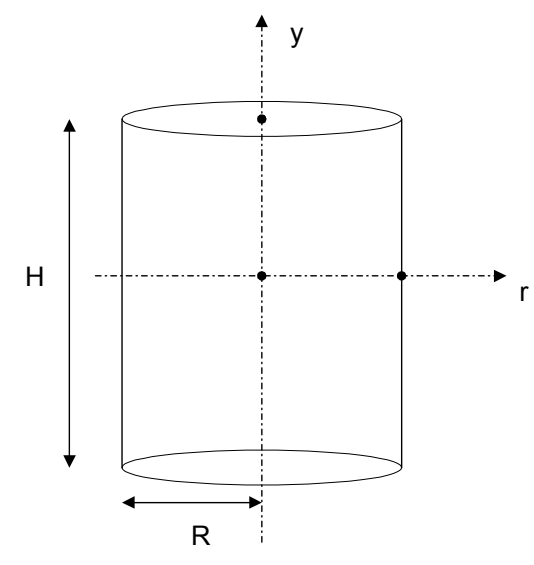

embalagem / produto alimentício.

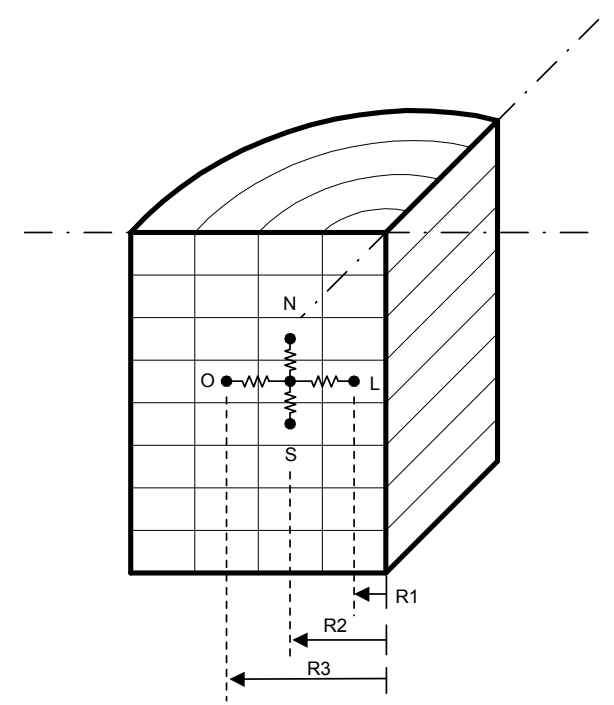

A capacitância térmica é calculada pela expressão $C=\ddot{I}$ 回 $\hat{\mathrm{a}}^{{ }^{\mathrm{TM}}} C_{p} \hat{\mathrm{a}}^{\wedge \mathrm{TM}} V_{I}$, e as condutâncias térmicas entre os nós podem ser de dois tipos: condutâncias radiais $\left(G_{I L}\right.$ e $\left.G_{I O}\right)$ e condutâncias axiais $\left(G_{I N}\right.$ e $\left.G_{I S}\right)$. Estas condutâncias são determinadas pelas Equações (3), (4) e (5), sendo que as Equações (3) e (4) representam exemplos típicos de condutâncias radiais e a Equação (5) de condutância axial.

$G_{I L}=\frac{\ddot{I} € \hat{\mathrm{a}}^{\wedge \mathrm{T}} K \hat{\mathrm{a}}^{\wedge \mathrm{TM}} d z}{2 \hat{\mathrm{a}}^{\wedge \mathrm{TM}} \ln \frac{R_{2}}{R_{1}}}$

$G_{I O}=\frac{\ddot{I} € \hat{\mathrm{a}}^{\wedge \mathrm{TM}} K \hat{\mathrm{a}}^{\wedge \mathrm{TM}} d z}{2 \hat{\mathrm{a}}^{\wedge \mathrm{TM}} \ln \frac{R_{3}}{R_{2}}}$

$G_{I N}=G_{I S}=\frac{\ddot{I} € \hat{\mathrm{a}}^{\hat{\top T M}} K \hat{\mathrm{a}}^{\hat{\top} \mathrm{TM}}\left(r_{e}^{2}-r_{i}^{2}\right)}{4 \hat{\mathrm{a}}^{\hat{T M}} d z}$

O sistema de equações gerado foi resolvido com o auxílio do código EES (Klein \& Alvarado, 2015).

\section{VALIDAÇÃO DO MODELO}

Devido à simetria das condições de contorno para o volume de controle a ser nodalizado, foi considerada a geometria formada por um setor circular de $90^{\circ}$ e de altura igual à metade da altura da embalagem. Foi gerada uma malha composta por 49 elementos de volume com 07 subdivisões na direção radial e 07 na direção axial. 
A validação deste modelo foi feita pela comparação dos resultados obtidos pela simulação computacional desenvolvida com base no modelo matemático proposto neste trabalho com aqueles apresentados por Gedraite (1999).

Na Figura 3 é apresentado o comportamento da temperatura da autoclave e uma comparação entre os resultados obtidos com o modelo matemático e o experimental.

\section{RESULTADOS \& DISCUSSÃO}

Na Figura 4 é apresentado o comportamento temporal das temperaturas do nó central $[1,7]$ para cinco valores diferentes da propriedade de transporte de calor difusividade térmica. Os valores da difusividade térmica foram escolhidos de maneira aleatória e utilizaram como referencia o valor igual a 2,0E-7 m²/s, obtido experimentalmente (Gedraite, 1999), com variação de amplitude entre os mesmos de $\pm 0,2 \mathrm{~m}^{2} / \mathrm{s}$.

O comportamento das temperaturas simuladas está adequado e coerente com o esperado, haja vista o fato de que com o aumento do valor da difusividade térmica, a variação de temperatura no ponto considerado tende a ser mais rápida.

Figura 3 - Comparação entre as temperaturas experimental e simulada do produto alimentício

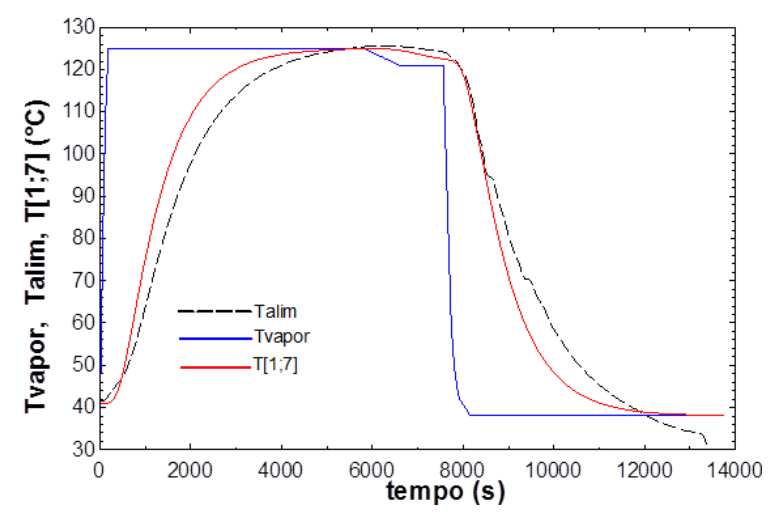

Figura 4 - Comparação entre as temperaturas simuladas do nó $[1,7]$ do produto alimentício

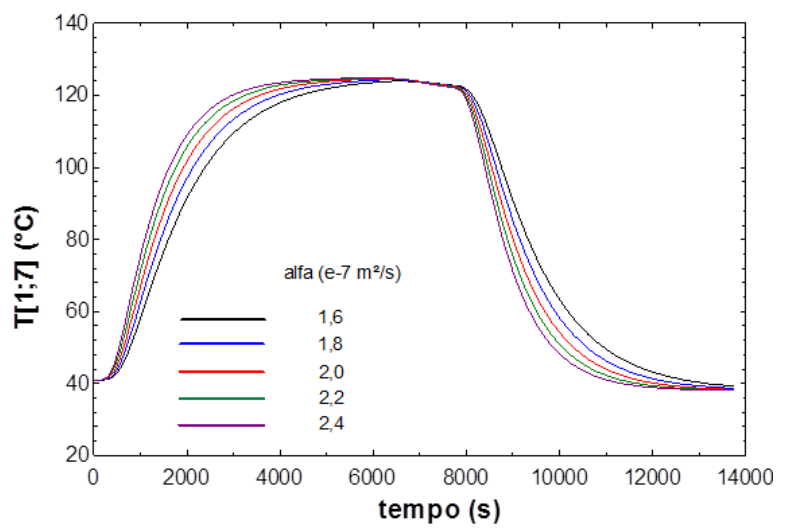

\section{CONCLUSÃO}

Observou-se que o modelo matemático representa, com boa aproximação, o comportamento da temperatura no interior do produto alimentício ensaiado.

O modelo matemático desenvolvido é adequado para uso na análise do processo de esterilização de produtos alimentícios que sejam aquecidos pelo mecanismo de transporte de calor por condução e apresentou tempo de execução pequeno e compatível com o requerido para uso em sistemas de automação de processos desta natureza. 


\section{NOMENCLATURA}

$\mathrm{dz}=$ Altura de um nó $(m)$

$\mathrm{C}_{\mathrm{p}}=$ Calor específico da PTS $\left(J / k g \hat{\mathrm{a}}^{\wedge \mathrm{TM}} \hat{\mathrm{a}}_{, \mathrm{f}}\right)$

$\mathrm{C}=$ Capacitância térmica $(J)$

$\mathrm{G}_{\mathrm{ij}}=$ Condutâncias térmicas entre os nós ( $m \hat{a}^{\wedge T M} \hat{a}, \mathrm{f} / W$ )

$\mathrm{K}=$ Condutividade térmica da PTS $\left(W / m \hat{a}^{\wedge T M} \hat{\mathrm{a}}, \mathrm{f}\right)$

$\mathrm{y}=$ Cota na direção axial $(m)$

$\mathrm{r}=$ Cota na direção radial $(m)$

$\rho=$ Densidade da PTS $\left(\mathrm{kg} / \mathrm{m}^{3}\right)$

$\alpha=$ Difusividade térmica da PTS $\left(\mathrm{m}^{2} / \mathrm{s}\right)$
$\mathrm{R}_{\mathrm{i}}=$ Raio correspondente ao i-ésimo nó $(m)$

$\mathrm{r}_{\mathrm{e}}=$ Raio externo do i-ésimo nó $(m)$

$\mathrm{r}_{\mathrm{i}}=$ Raio interno do i-ésimo nó $(\mathrm{m})$

$\mathrm{T}(\mathrm{t})=$ Temperatura da PTS no instante $\left(\hat{a}_{\mathrm{a}}, \mathrm{f}\right)$

$\mathrm{T}_{\mathrm{r}}=$ Temperatura de referência para a PTS $(\hat{a}, f)$

$\mathrm{T}=$ Temperatura do corpo $(\hat{\mathrm{a}}, \mathrm{f})$

$\mathrm{t}=$ Tempo de processamento $(s)$

$\mathrm{t}_{\mathrm{f}}=$ Tempo final de processamento $(s)$

$\mathrm{V}_{\mathrm{i}}=$ Volume do i-ésimo nó $\left(\mathrm{m}^{3}\right)$

\section{REFERENCIAS}

BASTOS, J. L. F. Notas de Aula da Disciplina Análise Termofluído Dinâmica de Reatores Nucleares. Depto. de Reatores do Instituto de Pesquisas Energéticas e Nucleares, 1994.

GEDRAITE, R. 1999. Desenvolvimento e Implementação de Algoritmo Computacional para garantir um determinado nível de Letalidade Acumulada para Micro-organismos presentes em Alimentos Industrializados. Escola Politécnica, Universidade de São Paulo, São Paulo-SP, Dissertação (Mestrado).

GEDRAITE, R., BASTOS, J. L. F., GARCIA, C. A., 1998. Utilização de modelagem matemática para otimizar o controle da temperatura de alimentos processados em autoclaves estacionárias, In Proc. of VIII Congresso Latino-americano de Controle Automático, Vinã del Mar-Chile, vol.2, pp. $679-683$.

GERMER, S. P. M. Avaliação dos desvios no processamento térmico de alimentos em conserva. Informativo FRUTHOTEC, v. 3, n. 2, p. 2-3, 1997.

KLEIN, S. A., ALVARADO, F. L. Engineering Equation Solver. Middleton, WI, 1996. Manual do Usuário, F-Chart Software.

TEIXEIRA, A. A. \& BALABAN, M. Computer Applications in Thermal Processing of Canned Foods. ITAL, Campinas, 1996. 